\title{
Simulation and Analysis on the Traffic Noise Models of Four-Lane Highway
}

\author{
MA Xin-tan ${ }^{1, a}$, FENG Shi-chang ${ }^{1, b^{*}}$ \\ ${ }^{1}$ Vehicles and Traffic Engineering, Henan University of Science and Technology, \\ Henan, China \\ amaxintan@163.com, ${ }^{\mathrm{b}}$ 1103444277@qq.com
}

\begin{abstract}
Keywords: Highway; 1/3OCT; Single source; Dual source; Simulation
Abstract. At present, highway traffic noise prediction models mostly use the equivalent continuous A-weighted sound pressure level to predict the noise within the country as well as in abroad, and they also usually use one line source to simulate the noise source in the simulation analysis of highway traffic noise, which makes some differences to the actual situation. This paper uses acoustic simulation software to establish a two-way four lane highway traffic noise model, and under single source prediction model and dual source prediction model, it calculates the sound pressure levels at four different receivers which are $20 \mathrm{~m}, 40 \mathrm{~m}, 80 \mathrm{~m}, 160 \mathrm{~m}$ distance from the road shoulder from frequency domain. Lastly, it analyses the sound pressure level frequency spectrum of the receivers under the two kinds of source models. Then came to a conclusion that, between the two kinds of source models in the two-way four lane highway model, the differences of the sound pressure levels at each center frequency of the receivers which are $20 \mathrm{~m}$ or far from shoulder, are not more than $1.29 \mathrm{~dB}$, and the differences of the total sound pressure levels are not more than $1.19 \mathrm{~dB}$. The conclusion will provide valuable references for the traffic noise control and prediction.
\end{abstract}

\section{Introduction}

Research Background. In recent years, the problem of traffic noise in our country is troubling the people on both side of the road, so it's very important to predict the traffic noise. At present, in the existing noise prediction models[1,2], most of them just only calculate the equivalent continuous A-weighted sound pressure level in the application process, and they're less defined the source from the aspect of spectrum. And the models usually only consider the effect of one line source to the sound pressure level of receivers, and rarely consider the effect of the road width to the definition of the source.

Usually, in the noise prediction, we commonly define a line source to simulate the sound source of the whole path at the road centerline or equivalent lane along the road direction, we call it single source model. It is distinct from the actual road condition [3], so it more accords with the actual situation of the road noise source when looking upon the road noise source as two lines sources, the predicted result is more accurate, and the two lines sources are called dual source model.

Main Work. In this paper, a two-way four lane highway model is established by using acoustic simulation software, and the single source model and dual source model are defined for simulation analysis. The frequency response curves of the sound pressure levels of four received points far away from the shoulders were calculated respectively. The simulation results of the two models in the frequency range of $63 \mathrm{~Hz}-4000 \mathrm{~Hz}$ were analyzed.

The traffic conditions on both sides of the central isolation belt are basically the same in the case of normal highway. Therefore, in this paper, the spectral characteristics of the two lines sources are supposed to the same. In the measurement, we just only need to measure one of the spectral characteristics of the two lines sources, and then the spectral characteristics of the total line source of the whole road can be obtained. In addition, it is difficult to measure the spectral characteristics of a single line sound source directly on the two-way four lane highway, so the method of analogous measurements can be used in practice [4]. The frequency response curve of the reference point which 
is $7.5 \mathrm{~m}$ distance from the equivalent lane can be measured according to the relevant standard. This curve can be used as preliminary data $[5,6]$.

\section{Simulated Analysis}

Four-lane Highway Model. The line sound source that was close to the received point is called "nearside lane line sound source", and the far one is called "farside lane line sound source". Four-lane highway model is shown in Fig. 1.

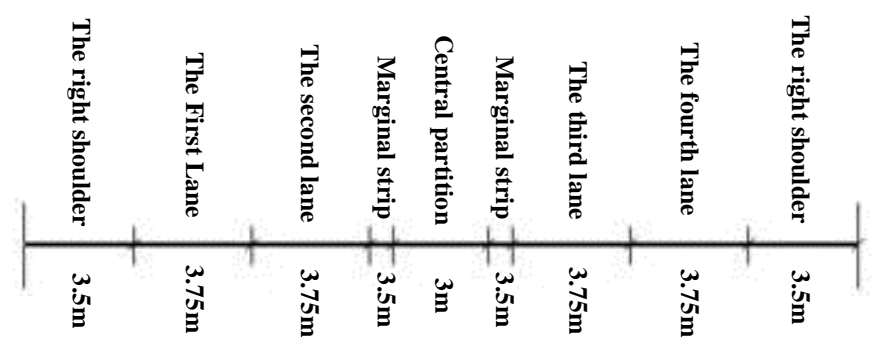

Fig. 1. The diagram of a four-lane highway

Analogous Measurements. According to analogous measurements, we need to find out a highway that has two lanes and is roughly the same with the unidirectional carriageway of a four-lane highway in traffic conditions, traffic flow, speed and environmental conditions. Then we require to set up the noise-measuring instrument at the position of the reference point which is $7.5 \mathrm{~m}$ distance from the equivalent lane, and measure the sound pressure level frequency spectrum of that highway, namely the spectrum is also the sound pressure level frequency spectrum of the reference point at the position of nearside lane line sound source and farside lane line sound source. The measurement results are shown in Table 1.

Tab.1 The sound pressure level spectrum of the line at $7.5 \mathrm{~m}$ distance equivalent traffic

\begin{tabular}{|c|c|c|c|c|c|c|c|c|c|c|}
\hline $1 / 3$ Octave[Hz] & 63 & 80 & 100 & 125 & 160 & 200 & 250 & 315 & 400 & 500 \\
\hline SPL[dB] & 56.02 & 65.23 & 63.57 & 75.72 & 75.98 & 79.33 & 78.27 & 80.88 & 80.53 & 80.98 \\
\hline $1 / 3$ Octave[Hz] & 630 & 800 & 1000 & 1250 & 1600 & $\mathbf{2 0 0 0}$ & $\mathbf{2 5 0 0}$ & $\mathbf{3 1 5 0}$ & $\mathbf{4 0 0 0}$ & \\
\hline SPL[dB] & $\mathbf{8 1 . 7 3}$ & $\mathbf{8 3 . 1 2}$ & $\mathbf{8 5 . 6 2}$ & $\mathbf{8 2 . 4 4}$ & $\mathbf{8 1 . 7 9}$ & $\mathbf{8 6 . 3 8}$ & $\mathbf{8 5 . 3 5}$ & $\mathbf{8 2 . 7 0}$ & $\mathbf{8 0 . 1 5}$ & \\
\hline
\end{tabular}

Theoretical Calculation. The software LMS.Virtual.lab usually uses sound power, sound power level or sound pressure to define the sound source, so the data in Table 1 needs to be transformed. In this paper, the data in Table 1 are converted to sound power level spectrum at the position of the sound source by using Eq. 1. Then we can get the sound power level spectrum of the single line sound source, as shown in Table 2.

$$
L_{w}=L_{p}-10 \lg \left(\frac{Q}{4 \pi}\right)
$$

Tab.2 sound power level spectrum of the single line sound source

\begin{tabular}{|c|c|c|c|c|c|c|c|c|c|c|}
\hline $1 / 3$ Octave[Hz] & 63 & 80 & 100 & 125 & 160 & 200 & 250 & 315 & 400 & 500 \\
\hline Sound Power Level $[\mathrm{dB}]$ & $\mathbf{6 7 . 7 8}$ & $\mathbf{7 6 . 9 9}$ & $\mathbf{7 5 . 3 3}$ & $\mathbf{8 7 . 4 8}$ & $\mathbf{8 7 . 7 4}$ & $\mathbf{9 1 . 0 9}$ & $\mathbf{9 0 . 0 3}$ & $\mathbf{9 2 . 6 4}$ & $\mathbf{9 2 . 2 9}$ & $\mathbf{9 2 . 7 4}$ \\
\hline $1 / 3$ Octave[Hz] & $\mathbf{6 3 0}$ & $\mathbf{8 0 0}$ & 1000 & 1250 & 1600 & $\mathbf{2 0 0 0}$ & $\mathbf{2 5 0 0}$ & $\mathbf{3 1 5 0}$ & $\mathbf{4 0 0 0}$ & \\
\hline Sound Power Level $[\mathrm{dB}]$ & $\mathbf{9 3 . 4 9}$ & $\mathbf{9 4 . 8 8}$ & $\mathbf{9 7 . 3 8}$ & $\mathbf{9 4 . 2 0}$ & $\mathbf{9 3 . 5 5}$ & $\mathbf{9 8 . 1 4}$ & $\mathbf{9 7 . 1 1}$ & $\mathbf{9 4 . 4 6}$ & $\mathbf{9 1 . 9 1}$ & \\
\hline
\end{tabular}

The received point is set at a distance of $20 \mathrm{~m}$ from the shoulder and $3 \mathrm{~m}$ above the ground in the model. According to the formula of equivalent lane position, as shown Eq. 2, the distance between the received point and the equivalent line of the third and fourth lane is $27.19 \mathrm{~m}$; The distance between the 
received point and the equivalent line of the first lane and the second lane is $39.21 \mathrm{~m}$; The distance between the received point and the equivalent line of the whole highway is $32.30 \mathrm{~m}$.

$$
r_{e q}=\sqrt{r_{\text {far }} \times r_{\text {near }}} \text {. }
$$

In the Eq. 2, $r_{e q}$ represents the distance between the receiver and the equivalent lane; $r_{f a r}$ represents the distance between the receiver and the centerline of the farthest lane; $r_{\text {near }}$ represents the distance between the receiver and the centerline of the nearest lane,

The data of Table 1 is also the spectrum of the reference point of the farside lane line sound source at the same time. According to the Eq. 3, we can calculate the spectrum that attenuates from the reference point of the farside lane line sound source to the reference point of the nearside lane line sound source, as shown in Table 3.

$$
L_{p}(r)=L_{p}\left(r_{0}\right)-10 \lg \left(\frac{r}{r_{0}}\right) .
$$

In the Eq. 3, $L_{p}(r)$ represents the sound pressure level of the received point; $L_{p}\left(r_{0}\right)$ represents the sound pressure level of the reference point.

Tab.3 Far lane line sound source close at the lane line sound source reference points of sound pressure level spectrum

\begin{tabular}{|c|c|c|c|c|c|c|c|c|c|c|}
\hline $1 / 3$ Octave[Hz] & 63 & 80 & 100 & 125 & 160 & 200 & 250 & 315 & 400 & 500 \\
\hline SPL[dB] & 51.87 & 61.08 & 59.42 & 71.57 & 71.84 & 75.18 & 74.12 & 76.73 & 76.38 & 76.84 \\
\hline $1 / 3$ Octave[Hz] & 630 & 800 & 1000 & 1250 & 1600 & 2000 & 2500 & 3150 & 4000 & \\
\hline SPL[dB] & 77.58 & 78.97 & 81.47 & 78.29 & 77.64 & $\mathbf{8 1 . 2 0}$ & $\mathbf{8 1 . 2 0}$ & 78.55 & 76.01 & \\
\hline
\end{tabular}

We can obtain the sound pressure level spectrum of the line sound source of the single source model at the reference point of the nearside lane line sound source by combining the sound pressure level spectrum of the reference point of the nearside lane line sound source and the sound pressure level spectrum of the reference point of the farside lane line sound source, as shown in Table 4.

Tab.4 The sound pressure level spectrum of the reference points in the simple sound source model

\begin{tabular}{|c|c|c|c|c|c|c|c|c|c|c|}
\hline $1 / 3$ Octave $[\mathrm{Hz}]$ & 63 & 80 & 100 & 125 & 160 & 200 & 250 & 315 & 400 & 500 \\
\hline SPL[dB] & 57.43 & 66.64 & 64.98 & 77.14 & 77.40 & 80.74 & 79.69 & 82.30 & 81.95 & 82.40 \\
\hline $1 / 3$ Octave[Hz] & 630 & 800 & 1000 & 1250 & 1600 & 2000 & 2500 & 3150 & 4000 & \\
\hline SPL[dB] & 83.14 & 84.54 & 87.03 & 83.85 & 83.20 & 87.79 & 86.77 & 84.11 & 81.57 & \\
\hline
\end{tabular}

According to geometrical relationship, the equivalent lane of the single source model is $12.62 \mathrm{~m}$ distance from its reference point. Then we need to convert the data in Table 4 into the sound power level spectrum by using the Eq. 1, as shown in Table 5 .

Tab.5 The spectrum sound power level of simple sound source

\begin{tabular}{|c|c|c|c|c|c|c|c|c|c|c|}
\hline $1 / 3$ Octave $[\mathrm{Hz}]$ & 63 & 80 & 100 & 125 & 160 & 200 & 250 & 315 & 400 & 500 \\
\hline Sound Power Level $[\mathrm{dB}]$ & 71.46 & $\mathbf{8 0 . 6 6}$ & 79.00 & 91.16 & 91.42 & 94.76 & $\mathbf{9 3 . 7 1}$ & 96.32 & 95.97 & 96.42 \\
\hline $1 / 3$ Octave[Hz] & 630 & $\mathbf{8 0 0}$ & 1000 & 1250 & 1600 & $\mathbf{2 0 0 0}$ & $\mathbf{2 5 0 0}$ & $\mathbf{3 1 5 0}$ & $\mathbf{4 0 0 0}$ & \\
\hline Sound Power Level $[\mathrm{dB}]$ & $\mathbf{9 7 . 1 6}$ & $\mathbf{9 8 . 5 6}$ & 101.05 & $\mathbf{9 7 . 8 7}$ & $\mathbf{9 7 . 2 2}$ & $\mathbf{1 0 1 . 8 1}$ & $\mathbf{1 0 0 . 7 7}$ & $\mathbf{9 8 . 1 3}$ & $\mathbf{9 5 . 5 9}$ & \\
\hline
\end{tabular}

Besides, in this paper, the model has also defined spectrum absorption properties of the ground and the environment factors such as temperature, humidity and barometric pressure. Fig. 2 shows the frequency response curve of the sound pressure level of the received point which is $20 \mathrm{~m}$ away from the shoulder in the single and dual source prediction models. Fig. 3 shows the difference between the single and dual sound sources at each center frequency in Fig. 2. The data in the graph shows that, for 
the two kind of models in the four-lane highway, in the frequency range of $63 \mathrm{~Hz}-4000 \mathrm{~Hz}$, the difference between the sound pressure levels at each center frequency of received points is between $0.56 \mathrm{~dB}$ and $1.29 \mathrm{~dB}$, the difference is very little. According to the formula of total sound pressure level, the total sound pressure level at received point of the single sound source model is $91.26 \mathrm{~dB}$, and the total sound pressure level at received point of the dual sound source model is $90.07 \mathrm{~dB}$, the difference between the two data is only $1.19 \mathrm{~dB}$, it's very small.

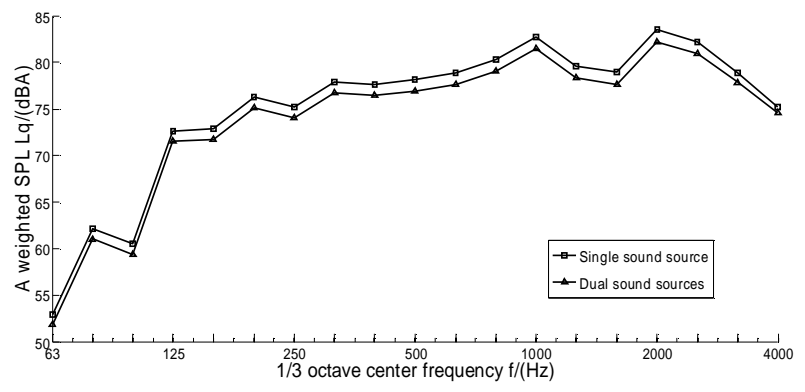

Fig. 2 The frequency response curve of the SPL of the received point which is $20 \mathrm{~m}$ away from the shoulder in the single and dual source prediction models

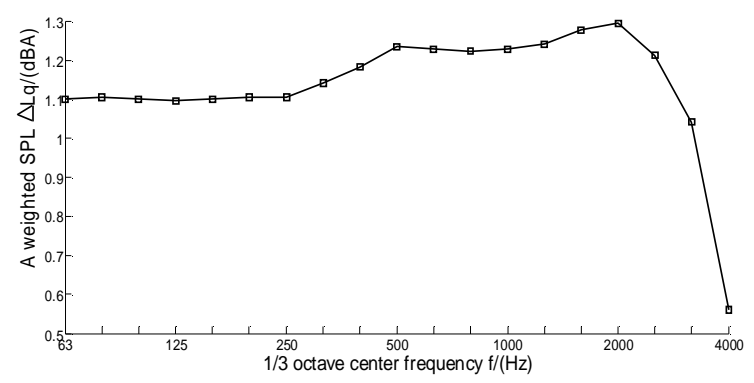

Fig. 3 The difference of each center frequency at 20m away from the shoulder in the two kinds of models

Expansion and Chapter Summary. Using the same method, we can calculate the difference of the sound pressure level at the position of the received point which is $40 \mathrm{~m}$ away from the shoulder between the two kinds of source models, the difference of the sound pressure level is between $0.41 \mathrm{~dB}$ and $0.59 \mathrm{~dB}$, and the difference of the total sound pressure level is $0.45 \mathrm{~dB}$; In the place of $80 \mathrm{~m}$, the difference of the sound pressure level is between $0.67 \mathrm{~dB}$ and $0.68 \mathrm{~dB}$, and the difference of the total sound pressure level is $0.67 \mathrm{~dB}$; In the place of $160 \mathrm{~m}$, the difference of the sound pressure level is between $0.67 \mathrm{~dB}$ and $0.68 \mathrm{~dB}$, and the difference of the total sound pressure level is $0.67 \mathrm{~dB}$. Thus it can be seen that the prediction of the sound pressure levels of the four received points by the two kinds of source models is not very different, the sound pressure level calculated by the single sound source is slightly higher than the sound pressure level calculated by the dual sound source, and with the increase of the distance, the overall trend of the total sound pressure level difference is reduced.

\section{Conclusions}

This paper uses acoustic simulation software to establish a two-way four lane highway traffic noise model, then simulates and analyses the difference of the sound pressure level spectrum from the frequency domain between the two kinds of source models, then comes to some conclusions as follows:

(1) In the single and dual sound source prediction models, the trend of the two spectral curves at the received points is kept well consistent, and the numerical difference of the two curves is small, which shows that the dual source model has certain feasibility and accuracy.

(2) For the four - lane highway, the single and dual sound source models both have good effect on the noise prediction, and with the increase of the distance between the received point and the sound source, the difference of the spectrum between the two sound source models at the position of the received 
points becomes smaller gradually, and the difference of the total sound pressure level becomes smaller gradually as well.

The above conclusions only apply to the four-lane highway. But for the highways which have more than four lanes, the effects of single and double sound sources on the sound pressure level of the received points need to study deeply.

\section{Acknowledgment}

This work was financially supported by the Modeling of Traffic Noise and Optimization of Acoustic Barrier(15A460019) and Outstanding Youth Plan for Science and Technology Innovation Talents in Henan Province(144100510004).

\section{References}

[1] The State Environmental Protection Administration Supervision and Management. China's Environmental Impact Assessment Training Materials [M]. Beijing: Chemical Industry Press, 2000. 269- 272.

[2] Cui Bao-jun, Tong Yi, Yan Ye-wei etc. Lane Highway Traffic Noise Prediction Model Selection Research [J]. China Water Transport: Second Half, 2012,(10):68-69.

[3] JTJ 005-96, Specifications for Environment Impact Assessment of Highway (on trial) [S]. 15- 16.

[4] Fan Dong-Ping, Road Traffic Noise Prediction Model Analysis and Empirical Research [D]. Guangdong University of Technology, 2012: 14-15.

[5] Xu Ling-lun. Highway Traffic Noise Prediction Model Application and Source of Inquiry [D]. Southwest Jiaotong University, 2011: 13-14.

[6] Wang Chuan-ying, Selection Analysis of Eight Lanes of Traffic Noise Prediction Model Based on FHWA [J]. Resources Economization and Environmental Protection, 2013, (3): 79-80. 